S3 Table. Acknowledgment of individuals and institutions who contributed data and valuable input to this project.

\section{Contact Name}

Shannon Albeke

Chad Anderson

Tara Anderson

Mark Balman

Lisa Baril

Joe Barnes

Carie Battisone

Greg Beatty

Dale Becker

Bryan Bedrosian

Allison Begley

Katie Benzel

David Bittner

Gretchen Blatz

Pete Bloom

Clint Boal

John Boone

Sandra Borthwick

Nick Brown

Kerry Burns

Melissa Burns

Tim Byer

Jason Carlisle

Jay Carlisle

John Carlson

Dan Casey

Ross Crandall

Miguel Cruz

Trish Cutler

Nathan Darnall

Keith Day

Jim Dick

Thomas Dietsch

Tom Dilts

Chris Dirk

Daniel Driscoll

Jamey Driscoll

Kathy Duttenhefner

Steve Dyke

April Estep

Tammy Fletcher

Thomas Flowers

Gail Garber

Rick Gerhardt

Sarah Haas

Derek Hall

Eric Hallingstad

Al Harmata

Rick Harness

Destin Harrell

Gerald Hayes

Gjon Hazard

Casey Heimerl

Highlands Ranch Community Association

Mary Jo Hill

Dylan Hopkins

Gregory Holm

Grainger Hunt
Contact Organization

University of Wyoming

National Park Service, Bryce Canyon National Park

Bureau of Land Managament, Shoshone Field Office

BirdLife International

National Park Service, Yellowstone National Park

Nevada Department of Wildlife

California Department of Fish and Wildlife

US Fish and Wildlife Service, Western Golden Eagle Team

The Confederated Salish and Kootenai Tribes

Teton Raptor Center

Montana Fish, Wildlife, and Parks

Bureau of Land Managament, Dillon Field Office

Wildlife Research Institute Inc

Washington Department of Fish and Wildlife

Bloom Biological, Inc

Texas Tech University, USGS Texas Cooperative Fish and Wildlife Research Unit Great Basin Bird Observatory

National Park Service, Capitol Reef National Park

NA

US Forest Service, Black Hills National Forest

US Fish and Wildlife Service, Utah Ecological Services Field Office

US Forest Service, Thunder Basin National Grassland

Western EcoSystems Technology, Inc

Intermountain Bird Observatory

Bureau of Land Managament, Montana/Dakotas State Office

Northern Great Plains Joint Venture

Craighead Beringia South

Pronatura Mexico

US Department of Defense, White Sands Missile Range

US Fish and Wildlife Service, Wyoming Ecological Services Field Office

Utah Division of Wildlife Resources

US Fish and Wildlife Service, Western Golden Eagle Team

US Fish and Wildlife Service, R8 Carlsbad Field Office

University of Nevada, Reno

North Dakota Parks and Recreation Department

American Eagle Research Institute

Arizona Game and Fish Department

North Dakota Natural History Inventory

North Dakota Game and Fish Department

Colorado Parks and Wildlife

US Forest Service, Caribou National Forest

Private Researcher

Hawks Aloft, Inc

SageScience

National Park Service, Bryce Canyon National Park

National Security Tehcnologies, LLC

Western EcoSystems Technology, Inc

Montana State University

EDM International

Bureau of Land Managament, Cody Field Office

Washington Department of Fish and Wildlife

US Fish and Wildlife Service, Western Golden Eagle Team

South Dakota Department of Game, Fish, and Parks

Highlands Ranch Community Association

US Fish and Wildlife Service, Charles M Russell National Wildlife Refuge

Utah State University, Department of Biology - Sullivan Lab

National Park Service, Grand Canyon National Park

The Peregrine Fund 
Rich Inman

Frank Isaacs

Kenneth Jacobson

Mackenzie Jeffries

Aran Johnson

Sandy Johnson

Kate Keiser

Kent Keller

John Kendall

Christy Klinger

David Klute

Sonya Knetter

Robbie Knight

Michael Kochert

Patrick Kolar

Patty Kruger

Rory Lamp

Russ Lawrence

David Leal

Karen Lee

Doug Leslie

Sarah Lindsey

Jim Lindstrom

Mike Lockhart

Julie Luetzelschwab

Robyn MacDuff

Erin Madson

Paige Maskill

Randy Matchett

Kyle McCarty

Trent McDonald

Gwyn McKee

Tony McKinney

Martin Miller

David Moen

Libby Mojica

Colleen Moulton

Robert Murphy

Robin Naeve

Randy Naugle

Janice Naylor

Justin Neighbor

Chris Nicolai

Russ Norvell

Brian Novosak

Bob Oakleaf

Wayne Peay

Kristen Philbrook

Lonnie Pilkington

Marty Piorkowski

Steve Plunkett

Katie Powell

Fritz Prellwitz

Chuck Preston

Louis Provencher

Drew Rayburn

Larry Reigel

David Rivers

Dan Roddy
US Geological Survey, Las Vegas Field Office

Oregon Eagle Foundation

Arizona Game and Fish Department

Nevada Department of Wildlife

Southern Ute Nation

North Dakota Game and Fish Department

California Department of Fish and Wildlife

Private Researcher

Bureau of Land Managament, Farmington Field Office

Nevada Department of Wildlife

Colorado Parks and Wildlife

Idaho Department of Fish and Game

US Army, Dugway Proving ground

US Geological Survey, Forest \& Rangeland Ecosystem Science Center

US Geological Survey, Forest \& Rangeland Ecosystem Science Center

USDA Forest Service, Pacific Southwest Region

Nevada Department of Wildlife

US Air Force, Hill AFB, UTTR

US Fish and Wildlife Service, Western Golden Eagle Team

US Forest Service, Rocky Mountain Regional Office

ICF International, Inc

Utah Natural Heritage Program

US Fish and Wildlife Service, Wyoming Ecological Services Field Office

Wildlands Photography and Bio-Consulting

US Forest Service, Sante Fe National Forest

Raptor Inventory Nest Surveys

US Fish and Wildlife Service Wyoming Ecological Services Field Office

US Fish and Wildlife Service Montana Ecological Services Field Office

US Fish and Wildlife Service, Charles M Russell National Wildlife Refuge

Arizona Game and Fish Department

Western EcoSystems Technology, Inc

Thunderbird Widllife Consulting, Inc

US Fish and Wildlife Service, R8 Carlsbad Field Office

Montana Natural Heritage Program

Nez Perce Wildlife Division

EDM International

Idaho Department of Fish and Game

US Fish and Wildlife Service, R2 Division of Migratory Birds

Bureau of Land Managament, Utah State Office

US Fish and Wildlife Service, Carlsbad Field Office

US Forest Service, Pawnee National Grassland

Red Cliffs Desert Reserve

US Fish and Wildlife Service, Reno Field Office

Utah Division of Wildlife Resources

Bureau of Land Management, Nevada

Wyoming Game and Fish Department (WGFD)

Raptor Inventory Nest Surveys (RINS)

US Forest Service, Pawnee National Grassland

National Park Service, Glen Canyon National Recreation Area

Arizona Game and Fish Department

US Forest Service, Southwestern Regional Office

US Fish and Wildlife Service, Western Golden Eagle Team

Bureau of Land Managament, Malta Field Office

Buffalo Bill Center of the West, Draper Natural History Museum

The Nature Conservancy

Jefferson County Open Space

US Fish and Wildlife Service, Oregon Fish and Wildlife Office

Native Range Capture Services

National Park Service, Wind Cave National Park 
Carl Rudeen

Matt Rustand

Robert Sacco

Amanda Schluter

Rachel Simpson

David Skinner

Steve Slater

Brian Smith

Chad Smith

Doug Smith

Jeff Smith

Kristina Smucker

Scott Somershoe

Rob Spaul

John Spence

Darci Stafford

Dale Stahlecker

John Stephenson

Janice Stroud-Settles

Brian Sullivan

Jason Sutter

Trish Sweanor

Bruce Thompson

Cris Tomlinson

David Topolewski

Nick Van Lanen

Chet VanDellen

Chris Vennum

Joe Vieira

Zach Wallace

Arden Warm

Jim Watson

Peter Weisberg

Bonnie Weller

Nathaniel West

Paul Whitefield

Lynn Wickersham

David Wiens

Gary Williams

Don Wolfe

Simon Wray

Dave Wrobleski

Mike Yates
Mountain Home Air Force Base

Bureau of Land Managament, Royal Gorge Field Office

Colorado Parks and Wildlife

Hawks Aloft, Inc

Nebraska Game and Parks Commission, Nebraska Natural Heritage Program

US Forest Service, Sawtooth National Forest

Hawkwatch International

US Fish and Wildlife Service, R6 Division of Migratory Birds

Navajo Nation

National Park Service, Yellowstone National Park

HT Harvey and Associates

Montana Fish, Wildlife, and Parks

US Fish and Wildlife Service, R6 Division of Migratory Birds

Boise State University

National Park Service, Glen Canyon National Recreation Area

Bureau of Land Managament, Buffalo Field Office

Eagle Environmental, Inc

National Park Service, Grand Teton National Park

National Park Service, Grand Canyon National Park

Cornell University, eBird

US Bureau of Land Management Idaho State Office

US Fish and Wildlife Service, Wyoming Ecological Services Field Office

Washington Department of Fish and Wildlife

Nevada Department of Wildlife

US Forest Service, Rio Grande National Forest

Rocky Mountain Bird Observatory

Nevada Department of Wildlife

Colorado State University

Bureau of Land Managament, Renewable Energy Office

Wyoming Natural Diversity Database

US Forest Service, Dakota Prairie Grasslands

Washington Department of Fish and Wildlife

University of Nevada, Reno

Nevada Department of Wildlife

Bureau of Land Managament, Tres Rios Field Office

National Park Service, Walnut Canyon National Monument

Fort Lewis College, San Juan Institute of Natural and Cultural Resources

US Geological Survey, Forest \& Rangeland Ecosystem Science Center

US Fish and Wildlife Service, Western Golden Eagle Team

University of Oklahoma, GM Sutton Avian Research Center

Oregon Dept of Fish and Wildlife

US Forest Service, Lolo National Forest

BioResource Consultants 